\title{
Publisher's Note: Disentangling the role of small polarons and oxygen vacancies in $\mathrm{CeO}_{2}$ [Phys. Rev. B 95, 245101 (2017)]
}

Lu Sun, Xiaowei Huang, Ligen Wang, and Anderson Janotti

(Q) (Received 14 February 2018; published 23 February 2018)

DOI: 10.1103/PhysRevB.97.079906

This paper was published online on 1 June 2017 with an incomplete labeling of a funding source in the Acknowledgments. On page 7, the first sentence of the Acknowledgments should read as "This work was supported by the National Science Foundation under Grant No. DMR-1652994.” The Acknowledgments have been corrected as of 15 February 2018. The Acknowledgments are incorrect in the printed version of the journal. 